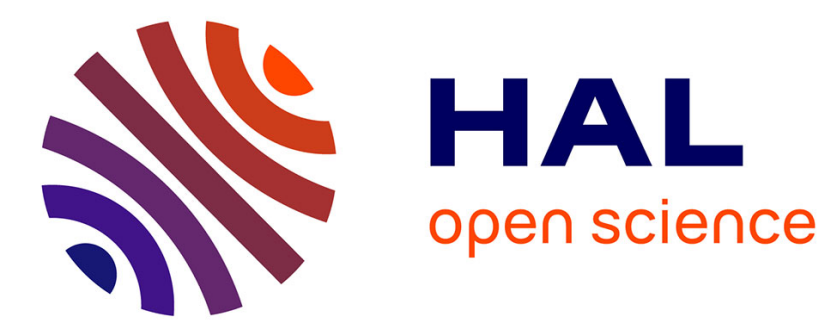

\title{
Entre santé et expérience de la maladie Janine Pierret
}

\section{To cite this version:}

Janine Pierret. Entre santé et expérience de la maladie. Psychotropes, 2008, 14 (2), pp.47-59. hal00337032

\section{HAL Id: hal-00337032 \\ https://hal.science/hal-00337032}

Submitted on 5 Nov 2008

HAL is a multi-disciplinary open access archive for the deposit and dissemination of scientific research documents, whether they are published or not. The documents may come from teaching and research institutions in France or abroad, or from public or private research centers.
L'archive ouverte pluridisciplinaire HAL, est destinée au dépôt et à la diffusion de documents scientifiques de niveau recherche, publiés ou non, émanant des établissements d'enseignement et de recherche français ou étrangers, des laboratoires publics ou privés. 


\section{Entre santé et expérience de la maladie}

\section{Janine Pierret}

Sociologue, directrice de recherche CNRS

CERMES (CNRS-INSERM-EHESS)

Site CNRS, 7 rue Guy Môquet, 94801 Villejuif cedex (France)

0149583689 pierret@vjf.cnrs.fr

Résumé : La place de la médecine dans la société et les rapports entre maladie et santé ont connu de profonds changements dans les dernières décennies. Le droit à la maladie et aux soins a été remplacé par le devoir de santé et il ne s'agit plus de lutter contre la maladie mais de se maintenir en santé. Avec l'émergence de la médecine du risque, un glissement s'effectue de l'évitement à l'anticipation des risques pour aller vers le toujours mieux, et bouleverse les repères entre santé et maladie. Ces transformations ont un impact sur la médecine elle-même et ses modalités de traitement et de suivi des personnes dont le statut entre santé et maladie est de plus en plus brouillé.

Mots-clés : santé, maladie, médecine, changements, prévention, risque, personne

\section{Between health and the experience of illness}

Abstract : The place of medicine in society and the relationships between illness and health have seen profound changes over recent decades. The right to be ill and to be treated has been replaced by the duty to be in good health and it is no longer a question of fighting illness, but rather of remaining healthy. With the emergence of risk medicine, we are seeing a shift from risk avoidance to risk anticipation in order to achieve the ever-better, thus losing our bearings between health and illness. These changes have impacted medicine itself and its ways of treating and monitoring people whose status remains blurred, somewhere between health and illness.

Key words: health, illness, medicine, changes, prevention, risk, person

Au cours des cinquante dernières années, la place de la médecine dans la société et les rapports entre santé et maladie ont connu de profondes transformations et de nombreux bouleversements. À partir des années 1950, on assiste à la montée en puissance de la médecine curative et à son emprise sur la société au point que l'on a pu parler de « médicalisation de la société » dans les années 1970. Simultanément, on a assisté à l'émergence de la conception globale de la santé qui ne se réduit pas aux seuls soins médicaux et qui dépasse l'institution médicale et les professionnels de soins. Elle implique de prendre en compte l'organisation sociale, la division du travail, les rapports sociaux, les politiques et les 
moyens existants et développés dans la société. La santé est alors un enjeu des politiques publiques et un bien collectif, mais elle est également devenue une valeur individuelle et même une norme dans notre société. Enfin, ces dernières années, on assiste à un déplacement et à une reconfiguration des rapports entre santé et maladie avec l'émergence du risque et de son anticipation avec le développement d'une médecine de surveillance.

Après avoir rappelé la montée en puissance de la médecine et la transformation de ses rapports avec la santé, seront ensuite interrogées les transformations récentes avec le développement d'une médecine de la santé dans une société du risque et l'accroissement du nombre de personnes qui vivent avec une maladie sans se considérer comme des malades.

La montée en puissance de la médecine : fascination et dénonciation de la médicalisation de la $\underline{\text { société }}$

Dès les années 1950, la logique à l'origine de l'essor de la médecine et du système de santé se fondait d'une part sur le souci reconnu d'autonomie des activités médicales et scientifiques et d'autre part sur la spécificité des modes de financement et de régulation des dépenses médicales. Sur ces deux pivots, l'un social et l'autre économique, s'est construit ce que l'on peut appeler une «délégation globale de légitimité » de la société à la médecine moderne pour la prise en charge des problèmes de santé. Au cours des années 1970, ce qui a été appelé la «médicalisation de la société» correspond à la montée en puissance de la médecine et à l'élargissement de son champ de compétences (Bozzini et al., 1981). Cet élargissement s'appuie à la fois sur le monopole de la thérapeutique et le contrôle des maladies, sur ses succès scientifiques, sur son idéologie humaniste et individualiste de lutte contre la souffrance et la mort au nom du bien du malade. Le savoir médical a pris une valeur normative par rapport à des secteurs de plus en plus nombreux de la vie individuelle, définis et évalués en termes de santé. L'alcoolisme, la maladie mentale, l'usage de drogue, les abus contre les enfants, sont passés d'une désignation morale ou criminelle à un registre médical. L'acte condamnable (badness) est devenu maladie (sickness) comme l'ont montré, sur la longue durée, les sociologues américains P. Conrad et W. Schneider (1985). La médecine est devenue une institution de contrôle social.

Transformer des vices, des crimes, des pêchés en maladie, pénétrer de plus en plus dans tous les aspects de la vie sociale - des différents âges de la vie à la conception à la mort -, imposer des règles alimentaires et une morale de la vie saine, plus rien n'échappe à la médecine et à la croyance que la médecine permet tout et peut tout régler ou arranger : 
permettre la vie quand cela est problématique, faire reculer les limites de la mort. Ainsi tout problème individuel mais aussi social peut, grâce aux avancées bio-médicales, recevoir une réponse technique adaptée. La bio-médecine derrière une apparente neutralité scientifique évacue les aspects moraux et normatifs de la maladie, de la vie et de la mort et surtout contribue à individualiser l'appréhension des problèmes en les rabattant sur « le malade ».

Pourtant, dès les années 1960-1970, des travaux d'historiens, de démographes, de statisticiens ont montré que la médecine curative avec ses moyens diagnostics et thérapeutiques de plus en plus sophistiqués, n'est ni la seule ni la principale responsable de l'amélioration de la santé et des gains de longévité réalisés. C'est au milieu des années 1970, qu'un virage se produit avec la «conception globale de la santé » avancée et développée au Canada (Lalonde, 1974). Elle s'appuie sur quatre éléments principaux : la biologie humaine, l'environnement, les habitudes de vie et l'organisation des soins de santé. Deux ans plus tard, cette conception est reprise en France dans le VIIème Plan qui constate que l'on est « entré dans une zone de rendements décroissants du point de vue de l'efficacité de la médecine ». Si la santé est mise en avant et renvoie au système de soins, elle implique aussi de prendre en compte l'environnement (c'est-à-dire l'ensemble des facteurs extérieurs au corps et qui échappent à la maîtrise de chacun) et les conditions et habitudes de vie de la population. Ainsi parler de santé, c'est aussi parler des politiques (de protection sociale mais aussi d'emploi, d'éducation, de logement et de soins, la médecine n'étant qu'un élément d'une structuration sociale de plus en plus complexe dans nos sociétés industrialisées et développées), des conditions de vie, des valeurs et des normes de cette société.

La santé est alors considérée comme un patrimoine collectif qu'il faut protéger et accroître en diversifiant les actions et un devoir de chacun vis-à-vis de lui-même et de la société. Chacun de nous est maître de sa propre santé et doit exercer sa responsabilité en la matière après y avoir été invité et formé grâce à une éducation sanitaire adaptée. La santé qui permettait de justifier les actions entreprises par la médecine curative sort de l'ombre pour devenir la valeur centrale, la norme de référence. Le problème ne serait plus tant de réparer un dysfonctionnement et de faire disparaître la maladie que de proposer à chacun de nouvelles habitudes de vie et l'adoption de comportements rationnels pour la santé (Pierret, 1984).

Le développement de la santé comme norme implique d'aider les gens à gérer leur vie selon des principes de prévention énoncés par la profession médicale comme si c'était la vie qu'il fallait guérir (Carpentier et al., 1980). Ce plaidoyer pour la responsabilité individuelle repose sur l'idée de la liberté des individus et de leur indépendance par rapport aux contraintes sociales. Le social est naturalisé et appréhendé comme une totalité qui s'impose à tous de 
façon uniforme sans prendre en compte la diversité des situations. Il est entendu qu'en informant chacun des risques qu'il encourt, on le met en demeure d'y résister. On est donc passé d'une conception du risque-maladie en termes d'assurance et de garantie a posteriori à une conception du risque de maladie en termes de cause et de responsabilité a priori et de culpabilisation de la victime (victim blaming) (Crawford, 1977).

À la guérison et la réparation des corps fait alors place le maintien de la santé et la prévention de la maladie. Au droit à la santé des années 1960 se substituent le devoir d'être bien portant et le devoir de santé. D'une médicalisation de la société, nous sommes passés à une « société du culte de la santé » (Zola, 1981) et à sa "santéisation ». "Supercatégorie normative » (Herzlich, 1984), la santé a donc changé de place : il ne s'agit plus tant de lutter contre la maladie que contre la mauvaise santé et de prévenir en réparant en amont et avant même l'apparition d'un dysfonctionnement, grâce aux possibilités techniques existantes. Sfez (1995) dénonce l'utopie de la santé parfaite comme nouvelle figure bio-écologique qui suggère l'idée d'une purification de la planète et de l'homme qui vise l'individu lui-même dans son existence.

Changements de la place de la médecine dans la société

Des transformations au sein de la médecine

En dépit d'avancées scientifiques incontestables, on assiste depuis une quinzaine d'années à une remise en question de la logique médicale, ce qui conduit à définir les termes d'un nouveau contrat entre société et médecine fondé sur les questions suivantes : quelle médecine ? pour quels hommes ? avec quels fondements intellectuel, social et moral ? à quels coûts ? dans le cadre de quelle société ? (Baszanger et al., 2002). Cette remise en cause peut être saisie à différents niveaux.

- Tout d'abord, des transformations internes à la médecine remettent en cause son homogénéité et son autonomie. Les disciplines médicales se diversifient et se multiplient avec le développement des connaissances et des techniques, et la distance s'accroît entre celles qui sont fortement liées à la recherche scientifique et clinique et celles qui sont confrontées aux transformations démographiques et sociales (gérontologie, toxicomanie...). L'autonomie de la profession médicale est également bousculée avec l'accroissement des professionnels concernés qui interviennent dans des lieux différents et de plus en plus nombreux: les réseaux, les centres de jour, les lieux de vie, les soins à domicile... Le nombre, la qualification et le statut des professionnels impliqués auprès d'un même malade tout au long de son histoire se sont diversifiés. Si les lieux d'échanges entre médecins, intervenants divers et 
malades se sont démultipliés, en ville comme à l'hôpital, la question de la prise en charge des malades et de leur information devient centrale. Parmi ces groupes professionnels aux cultures et aux modalités d'intervention différentes qui prendra en charge l'information du malade, où se situeront les responsabilités de chacun, comment décider de la nature et de l'étendue de l'information à donner au malade ? La question de l'information, de l'éducation et de la responsabilisation devient une préoccupation centrale et ce d'autant que le développement d'associations de malades et d'usagers aux objectifs pouvant aller de l'aide pour la vie quotidienne à l'action politique, conduit à s'interroger sur son impact et ses effets. La mise en place de ces nouveaux rapports, en particulier avec la loi sur le droit des malades de 2002, n'est pas exempte de risques de judiciarisation dans la santé comme cela est déjà le cas OutreAtlantique.

- Ensuite, l'autonomie de la médecine et des professions médicales est également remise en cause avec le développement de la santé publique qui transforme la façon de poser les questions de santé et d'y répondre. L'augmentation du nombre des professionnels autorisés à prendre la parole se traduit par l'affaiblissement du pouvoir des experts attitrés et bien identifiés. On assiste alors au développement de prises de paroles hétérogènes et à des configurations variables entre acteurs selon les problèmes (médecins, scientifiques, politiques, médias, industriels, malades ...) (Baszanger et al., 2002). Les crises de santé publique sont des exemples particulièrement éclairants de cette multiplication et de cette superposition d'avis relançant continûment le débat et maintenant une forte incertitude dans un contexte où le risque zéro est évoqué voire invoqué comme référence improbable et même impossible à atteindre : l'affaire du sang contaminé ou de la transmission du prion. La sécurité sanitaire en réponse à des risques issus de l'alimentation, de l'environnement et du système de soins est devenue un sujet de préoccupation majeur dans nos sociétés démocratiques développées et conduit à s'interroger sur la démocratie sanitaire et de nouvelles formes de gouvernance.

- Enfin, les échecs successifs des procédures de maîtrise des dépenses interrogent la logique d'exception financière. De nouveaux outils d'allocation et de gestion des ressources et des modes inédits de régulation sont au cœur de la définition des politiques de santé. Des débats portent sur les méthodes d'évaluation des thérapies et plus largement des soins : comment définir et développer des bonnes pratiques de soins ? comment mesurer et comparer l'activité des services hospitaliers ? les indicateurs de qualité couramment utilisés (volume d'activité, mortalité hospitalière, attractivité de l'établissement, durée moyenne de séjour, taux de césariennes et d'infections nosocomiales) sont-ils toujours valides et suffisants ? En France, c'est à partir de 1996, avec la maîtrise médicalisée des dépenses de santé que le souci 
d'avoir des établissements hospitaliers performants s'est imposé et a conduit au développement de la démarche qualité à travers des procédures d'accréditation par des audits externes volontairement demandés par les établissements de soins (Moisdon, 2002 ; Minvielle, 2006). La question de la performance associe aux mesures de productivité, de l'évaluation de la qualité des services rendus et des coûts, un questionnement sur la place des usagers (Lombrail et al., 1999 ; Bachimont et Letourmy, 2002 ; Minvielle et al., 2008).

\section{D'une médecine de la maladie à une médecine du risque}

La santé publique se développe de façon parallèle et même en dehors de la médecine clinique et du savoir médical, avec la mise en place d'une approche des problèmes radicalement différente. Elle mobilise des connaissances multiples comme l'épidémiologie, les biostatistiques, le droit et les sciences sociales pour aborder les questions de santé en termes populationnels et probabilistes avec la notion de « facteur de risque » (Berlivet, 2005). Au cours des années 1970, la lutte contre le tabac et le cancer avait contribué à faire connaître cette approche, mais c'est avec l'irruption du VIH/sida, dans la première moitié des années 1980, que s'est diffusée et fait connaître du grand public la notion de «facteur de risque ». Cette notion aujourd'hui largement utilisée a connu des dérives et a donné lieu à des confusions avec les notions de groupes à risque ou de comportements à risque et surtout des glissements entre risque et cause, entre raisonnement probabiliste et raisonnement causal (Herzlich et Pierret, 1998).

Nos sociétés occidentales qui ont des conditions de vie de plus en plus sûres connaissent, simultanément et de façon paradoxale, une hypersensibilité aux risques. Et pourtant, ces dernières années, des affaires comme le sang contaminé ou la vache folle auront provoqué infiniment moins de victimes que les famines ou les épidémies des siècles passés ou même que les accidents de la route ou du travail aujourd'hui. Cela a pu se traduire par une relative remise en cause de l'idée de progrès et à des interrogations sur la science elle-même qui apparaît démunie face à certains risques, voire même perçue comme l'une des sources de ces derniers. En effet, des catastrophes écologiques ou technologiques (marées noires, accidents nucléaires...) n'auraient pas eu lieu sans les avancées scientifiques et techniques. Par ailleurs, parler en termes de risque vient à la fois révéler et accélérer le processus de laïcisation des sociétés modernes car l'adoption d'une perspective rationnelle et probabiliste rend le recours au destin et à la fatalité caduque. Parallèlement, se développe la croyance que la technique peut tout et que l'on peut agir techniquement pour réduire «tous » les risques 
quel que soit le domaine d'activité (système de sécurité d'alarme, de prévision météorologique...).

Aujourd'hui, l'intérêt des scientifiques se déplace de plus en plus vers des événements qui se situent aux limites de l'observable (faibles doses, effets à long terme, nouveaux risques infectieux) et des problèmes sont soulevés sur la base de simples hypothèses. Nos sociétés sont de plus en plus prises dans des tensions entre « en faire trop » avec les risques de dérives « sanitaires sécuritaires » et «ne rien faire » qui demande un travail d'explication et de réassurance qui n'est pas toujours suffisant. L'exemple de la vache folle en France illustre cette difficulté des pouvoirs publics pris entre l'urgence de répondre et de faire quelque chose, de ne pas contribuer davantage à l'affolement, et de satisfaire à la fois producteurs et consommateurs.

Des travaux ont également montré que la perception du risque est socialement sélective et renvoie aux positions, aux pratiques dans les institutions et aux expériences subjectives et sociales des individus. Cela signifie que les risques sont évalués en fonction des décisions dans lesquelles chacun est engagé et non en fonction d'une rationalité externe qui évaluerait les aspects négatifs et positifs indépendamment de tout contexte social ou de toute expérience sociale. Enfin, l'appréciation du risque ne se fait pas seulement au nom d'une logique de santé ou de prévention, mais prend en compte d'autres logiques, familiales ou économiques, c'est-à-dire que le « souci de soi »n'est pas toujours l'objectif primordial mais qu'il est pris dans un ensemble de contraintes et de contradictions qui contribuent à orienter la décision. Il s'agit alors de prendre la décision «la moins mauvaise possible », dans un contexte d'incertitude et parfois d'absence même de solution.

\section{De nouveaux rapports entre normal et pathologique, entre santé et maladie?}

Une « société du risque » (Beck, 1992 ; Giddens, 1994) caractérisée par l'anticipation des risques et la promotion du dépistage que viendrait renforcer le développement de la génétique et de la médecine prédictive ne serait-elle pas en train de se développer ? À la fin des années 1970, le modèle de la prévention consiste à lutter contre l'apparition de certaines $\underline{\text { maladies }}$ en intervenant auprès de membres de sous-groupes que l'on repère soit au niveau spatial, soit à travers leurs comportements en vue de favoriser leur intégration sociale (catégories défavorisées de certains quartiers, fumeurs, individus qui ont du cholestérol...). Déjà en 1981, Castel insistait sur le fait que : «L'éventuelle intervention vise des individus présélectionnés sur des critères individualisés, décontextualisés par rapport à l'environnement et économise une action préventive générale sur le milieu ». 
Aujourd'hui, le modèle de la prévention et du dépistage vise plutôt à améliorer et à

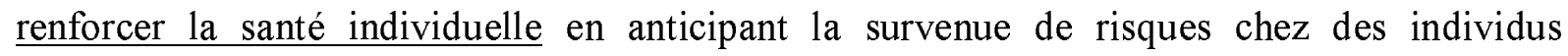
sélectionnés uniquement sur la base de quelques critères biologiques ou biologisés ou des phases de la vie comme l'âge et le sexe (femmes entre 50 et 74 ans, personnes issues de familles dans lesquelles un cancer de l'intestin a été diagnostiqué, ménopause...). Avec le développement du dépistage génétique, une dimension supplémentaire est introduite : les risques ne sont plus externes et liés aux comportements individuels ou aux conditions de vie, mais sont dans l'individu lui-même. Ne risque-t-on pas d'assister à un renversement de point de vue qui, sur la base du chacun pour soi, contribuerait à accroître l'individualisme et à affaiblir les liens sociaux ? On peut s'interroger sur le développement de cette «médecine de surveillance » (Armstrong, 1995) qui est de plus en plus anticipatrice et qui remet fondamentalement en question son seulement les rapports entre normal et pathologique, entre santé et maladie mais aussi l'identité des individus.

On sortirait donc d'un modèle de l'évitement des risques pour entrer dans un modèle du «toujours mieux» comme l'illustre la longue liste des interventions médicales sur les biens portants (procréation médicalement assistée, chirurgie esthétique, changement de sexe, lutte contre le vieillissement...). Ainsi à reculer les barrières du possible, le système de soins et la médecine vont devoir gérer leurs propres excès et leurs propres manques. La maladie en n'étant plus une fatalité mais une production dont l'individu serait responsable peut conduire à lui demander de modifier son présent en vue d'un futur plus raisonnable ou meilleur. La « médecine de la santé » introduit une nouvelle normalité qui ne serait plus biologique mais comportementale et un déplacement normatif serait entrain de s'opérer du côté de l'adaptation des comportements (Golse, 2001). Cela pourrait impliquer pour le système de soins de développer d'autres modalités de prises en charge, par exemple comment faire avec un individu qui se sait porteur sain d'une maladie dont personne ne sait ni quand ni comment elle va se déclarer. Et en s'affranchissant de la maladie, la médecine deviendrait un savoir total sur l'homme et quitterait le biologique pour demander au psychologique et au social de rendre compte du biologique. La médecine va-t-elle devenir une science de l'information sur l'individu, avec un savoir de plus en plus individualisant et décontextualisé ?

\section{Un nouveau personnage?}

Ces transformations en cours ne doivent pas faire oublier les progrès thérapeutiques réalisés, le recul de la mortalité dans certaines maladies et le développement de maladies de longue durée. Les recherches en sciences sociales ont montré que ces maladies ne se vivaient 
pas seulement dans le monde médical mais qu'elles affectaient l'ensemble des rapports avec l'entourage familial, amical et même professionnel et qu'elles pouvaient modifier la biographie des personnes atteintes (Pierret, 2003). Un nombre de plus en plus élevé de personnes vivent avec des pathologies lourdes, invalidantes et même mortelles (infection à VIH par exemple). Ces maladies avec lesquelles elles vont vivre de nombreuses années laissent apparaître un nouveau personnage qui n'est ni malade, ni en bonne santé. Il s'agit de personnes atteintes d'une maladie parfois mortelle et qui vivent avec un traitement quotidien et/ou ont un suivi médical régulier au long cours. Elles ne se considèrent pas comme des malades et entendent avoir une vie normale et/ou mener une vie la plus normale possible (Pierret, 2006). Que dire des personnes qui prennent des traitements de substitution aux opiacés : sont-elles maintenues dans une dépendance? sont-elles devenues abstinentes ? La maladie se vit de plus en plus sur un mode séquentiel et intermittent avec des consultations médicales et des examens complémentaires ou même des épisodes d'hospitalisation, autant de moments qui viennent rappeler sa présence

Certaines personnes assument et même revendiquent cette situation qui par ailleurs fait écho aux injonctions à l'autonomie et à la responsabilité individuelle diffusées par les politiques et les professionnels de santé. Pour d'autres qui sont dans des situations plus instables et difficiles sur le plan de la formation, de l'emploi ou de l'insertion sociale et professionnelle, le fait qu'elles soient reconnues comme des malades leur donne un statut social (être malade est socialement mieux perçu qu'être chômeur ou usager de drogue) et permet même l'ouverture et l'accès à certains droits sociaux (ALD, AAH et APL). Ce sont autant de situations qui devraient pouvoir coexister dans une société mais actuellement tout semble se passer comme si le modèle de la responsabilité individuelle et de l'autonomie primait dans la maladie comme dans la santé.

Inversement, une personne en parfaite santé peut être suivie régulièrement par la médecine soit parce qu'elle a eu plusieurs années auparavant une pathologie lourde (cancer, infarctus...) soit parce qu'elle est dans un modèle du « toujours mieux » et de l'anticipation des risques. Ces situations conduisent à insister sur le fait qu'une personne peut consulter et être suivie sur le plan médical sans être malade et qu'une personne atteinte d'une maladie ne se vit pas en permanence comme malade sauf dans des moments particuliers. D'autant que la sociologie de la maladie a toujours refusé de rabattre la maladie sur la personne et de parler de tuberculeux, de cancéreux ou d'hémophile. L'accent est mis sur la personne qui est atteinte d'une maladie avec laquelle elle vit. C'est pourquoi il s'agit de s'interroger sur les termes employés et de se demander pourquoi et quand parler de patient ou de malade. Rappelons que 
les associations de lutte contre le sida ont imposé, dès 1984, People with Aids repris en français par «personne avec le sida » qui s'est ensuite transformé en « personne vivant avec le VIH/sida ». Mais quel que soit le terme employé, personne, sujet, usager, consommateur, client il est important que les personnes concernées s'y reconnaissent et qu'une étiquette ne leur soit pas imposée de l'extérieur.

\section{En guise de conclusion}

$\mathrm{Au}$ cours des années 1970, plusieurs mouvements sociaux se sont élevés contre l'imposition des valeurs médicales et la main mise des professionnels sur la vie des personnes en dénonçant la médicalisation de la société. À la même époque, la santé devenait une valeur centrale et même la norme de référence. Depuis une vingtaine d'années, ce que recouvre la maladie et la santé comme valeur sont prises dans des tensions et font l'objet d'injonctions de plus en plus contraignantes. Aujourd'hui, les transformations dans les capacités d'intervention de la médecine, dans la nature des pathologies, les déplacements des frontières entre le médical et le social, le rôle des mouvements de consommateurs et des associations de malades, ont permis une relative autonomie des personnes vivant avec des maladies de longue durée. Parallèlement, des membres des catégories sociales moyennes et supérieures sont de plus en plus acquis à la valeur santé et à l'importance d'être responsable de leur capital santé (Pierret, 1984). Ils vont alors adopter des comportements hygiéniques, surveiller leurs consommations et même consulter régulièrement et de façon préventive un médecin pour conserver leur santé et pas seulement pour éviter la survenue d'une maladie. Simultanément, les pouvoirs publics ont tendance à se désengager en matière de dépenses de soins (franchises médicales) et l'accès aux divers services devient plus restrictif et difficile. Et de façon paradoxale, on pourrait se demander si on a encore le droit d'être malade aujourd'hui.

Ces changements qui s'amorcent et qui reposent la question des rapports entre la médecine et le corps social ne doivent pas pour autant nous faire oublier la persistance d'inégalités sociales dans la santé : inégalités sociales révélées par le système de soins ou accentuées par lui ? la santé comme marqueur du social ou l'inverse ? Comme le rappellent les auteurs d'un ouvrage sur les inégalités (Leclerc et al., 2000) parler en termes d'inégalités c'est reconnaître que la société en est à l'origine et cela implique de se situer d'emblée à ce niveau et non à celui d'une catégorie particulière nommée selon les époques, "pauvres », « précaires », « vulnérables ». Les inégalités mettent en cause les différentes composantes de la société et leur réduction suppose des interventions sur l'ensemble de la structure sociale et 
ne relève pas de la seule médecine et des politiques de santé car 30\% des inégalités seraient dues au système de soins. Mais constater par exemple, que les disparités de mortalité subsistent entre hommes et femmes et entre catégories socio-professionnelles, en France comme dans la plupart des pays, ne conduit pas pour autant à identifier une ou des explications. Disparités qui renvoient à des inégalités sociales plus fondamentales en termes de place dans l'organisation sociale et de conditions de vie, d'inégalités face à l'éducation, au logement et au travail ainsi que dans les valeurs et les références. Par exemple, le « souci de soi » suppose de pouvoir se projeter dans l'avenir. Est-il possible d'avoir une vision à long terme quand il faut parer au plus pressé et que la réalité quotidienne impose de vivre au jour le jour?

\section{$\underline{\text { Références bibliographiques }}$}

Armstrong D : « The rise of surveillance medicine » - In Sociology of Health and Illness, 17 (3) : 393-404 (1995)

Bachimont J, Letourmy A : «L'élaboration d'indicateurs de la performance hospitalière : quels enjeux pour les usagers ?»-In Sciences Sociales et Santé, 20 (2) : 105-115 (2002)

Baszanger I, Bungener M, Paillet A : Quelle médecine voulons-nous ? - Paris, La Dispute (2002)

Beck U : Risk society. Toward a new modernity - London, Sage Publications (1992)

Berlivet L: «Exigence scientifique et isolement institutionnel: l'essor contrarié de 1'épidémiologie française dans la seconde moitié du XXè siècle »-In Jorland G, Opinel A, Weisz G (sous la direction de) : Body Counts. Medical quantification in historical and sociological perspectives. La quantification médicale, perspectives historiques et sociologiques - Montréal et Kingston, Queen's University Press, 335-358 (2005)

Bozzini L, Renaud M, Gaucher D, LLambias-Wolff J (sous la direction de) : Médecine et Société. Les années 80 - Québec, Éditions coopératives Albert Saint-Martin (1981)

Carpentier J, Castel $\mathrm{R}$, Donzelot $\mathrm{J}$ : Résistances à la médecine et démultiplication du concept de santé, CORDES, rapport de recherche (1980)

Castel R : La gestion des risques - Paris, Éditions de Minuit (1981)

Conrad P, Schneider W: Deviance and medicalization. From badness to sickness Colombus, Merrill Publishing Company (1985)

Crawford $\mathrm{R}$ : «C'est de ta faute : l'idéologie de la culpabilisation de la victime et ses applications dans les politiques de santé » - In Bozzini L, Renaud M, Gaucher D, LLambias- 
Wolff J (sous la direction de): Médecine et Société. Les années 80 - Québec, Éditions coopératives Albert Saint-Martin, 481-512 (1981)

Giddens A : Les conséquences de la modernité - Paris, L'Harmattan (1994)

Golse A : « De la médecine de la maladie à la médecine de la santé 》-In Artières P, Da Silva E (sous la direction de) : Michel Foucault et la médecine. Lectures et usages - Paris, Éditions Kimé, 273-300 (2001)

Herzlich C : «Médecine moderne et quête de sens : la maladie signifiant social »- In Augé M, Herzlich C (sous la direction de) : Le sens du mal. Anthropologie, histoire et sociologie de la maladie - Paris, Éditions des Archives Contemporaines, 189-215 (1984)

Herzlich C, Pierret J : «Une maladie dans l'espace public. Le SIDA dans six quotidiens français » - In Annales E.S.C., n5, Septembre-Octobre, 1109-1134 (1988)

Lalonde M : Nouvelle perspective de la santé des Canadiens - Ottawa, Éditeur officiel (1974) Leclerc A, Fassin D, Grandjean H, Kaminski M, Lang T : Les inégalités sociales de santéParis, Éditions La Découverte (2000)

Lombrail P, Naiditch M, Baubeau D, Cuneo P: «Les éléments de la 'performance hospitalière'. Les conditions d'une comparaison »-DREES, Études et résultats, ${ }^{\circ} 42,8 \mathrm{p}$. (1999)

Minvielle $\mathrm{E}$ : «Les critères de qualité en médecine : jusqu'où aller?»-In Annales d'Urologie, 40, 3, 184-191 (2006)

Minvielle E, Sicotte C, Champagne F, Contandriopoulos AP, Jeantet M, Préaubert N, Bourdille A, Richard C: " Hospital Performance: Competing or Shared Values? »- In Health Policy, 87, 8-19 (2008)

Moisdon J C : «L'hôpital entre permanences et mutations » In Baszanger I, Bungener M, Paillet A (sous la direction de), Quelle médecine voulons-nous? - Paris, La Dispute, 35-54 (2002)

Pierret J : «Les significations sociales de la santé. Paris, l'Essonne, l'Hérault »-In Augé M, Herzlich C (sous la direction de) : Le sens du mal. Anthropologie, histoire et sociologie de la maladie - Paris, Éditions des Archives Contemporaines, 217-256 (1984)

Pierret J : « The illness experience: State of knowledge and perspectives for research »- In Sociology of Health and Illness, vol. 25, silver anniversary issue, 4-22 (2003)

Pierret J : Vivre avec le VIH. Enquête de longue durée auprès des personnes infectées - Paris, PUF, Collection Le lien social (2006)

Sfez L : La santé parfaite. Critique d'une nouvelle utopie - Paris, Le Seuil (1995) 
Zola I K : «Culte de la santé et méfaits de la médicalisation» In Bozzini L, Renaud M, Gaucher D, LLambias-Wolff J (sous la direction de) : Médecine et Société. Les années 80 Québec, Éditions coopératives Albert Saint-Martin, $31-51$ (1981) (version anglaise, 1977) 